\title{
Karin HAUSEN (dir.), Geschlechtergeschichte als GesellschaftsgeschichteGeschlechtergeschichte als Gesellschaftsgeschichte
}

Göttingen, Vandenhoeck \& Ruprecht, coll. « Kritische Studien zur Geschichtswissenschaft », 2012

\section{Sarah Baumann}

\section{(2) OpenEdition}

Journals

Édition électronique

URL : http://journals.openedition.org/clio/11794

DOI : $10.4000 /$ clio. 11794

ISSN : 1777-5299

Éditeur

Belin

Édition imprimée

Date de publication : 31 décembre 2013

ISSN : 1252-7017

Référence électronique

Sarah Baumann, « Karin hausen (dir.), Geschlechtergeschichte als

GesellschaftsgeschichteGeschlechtergeschichte als Gesellschaftsgeschichte », Clio. Femmes, Genre, Histoire [En ligne], 38 | 2013, mis en ligne le 08 janvier 2014, consulté le 22 septembre 2020. URL http://journals.openedition.org/clio/11794; DOI : https://doi.org/10.4000/clio.11794

Ce document a été généré automatiquement le 22 septembre 2020.

Tous droits réservés 


\section{Karin HAUSEN (dir.), \\ Geschlechtergeschichte als \\ GesellschaftsgeschichteGeschlechtergeschichte als Gesellschaftsgeschichte}

Göttingen, Vandenhoeck \& Ruprecht, coll. « Kritische Studien zur

Geschichtswissenschaft », 2012

Sarah Baumann

\section{RÉFÉRENCE}

Karin HAUSEN (dir.), Geschlechtergeschichte als Gesellschaftsgeschichte, Göttingen,

Vandenhoeck \& Ruprecht, coll. « Kritische Studien zur Geschichtswissenschaft », 2012, $394 \mathrm{p}$.

\section{NOTE DE L'ÉDITEUR}

Traduit de l'allemand par Tiphaine Robert

Karin Hausen compte parmi les pionnières de la recherche en histoire des femmes et du genre. Son nouvel ouvrage Geschlechtergeschichte als Gesellschaftsgeschichte (Histoire du genre comme histoire sociale) offre un choix cohérent de textes, pour la plupart déjà publiés, qui ne se bornent pas à documenter la carrière intellectuelle de l'historienne mais qui invitent à repenser «l'histoire du genre comme champ d'expérimentation scientifique et comme discipline historique sous l'angle de ses continuités, de ses approfondissements et de sa pertinence actuelle » (p. 15). La combinaison entre le parcours de recherche de la chercheuse et les développements historiographiques du 
genre donne un attrait tout particulier à ce livre, qui s'adresse autant aux étudiant-e-s qu'aux professionnel-le-s des sciences historiques et sociales.

Dans son introduction, Karin Hausen évoque les enjeux historiographiques du genre. La recherche en histoire des femmes et du genre s'est développée à une époque où les sciences historiques s'émancipaient de la prééminence du politique et se reformulaient dans une histoire des sociétés étoffée par l'histoire économique et sociale et par l'histoire des mentalités. Bien que l'histoire du genre ait participé à cette redéfinition, elle se trouva rapidement cantonnée à la marge des sciences historiques. Placer les femmes sous la loupe historiographique et intégrer la catégorie genre dans les théories dominantes équivalait à remettre en question les fondements des travaux historiques effectués jusqu'alors. Entre ces pôles de recherche - souvent jugés inconciliables Karin Hausen joua un rôle clé dans les débats scientifiques germanophones. Pendant plusieurs décennies, son travail a imprégné l'histoire sociale et économique de l'Allemagne tout en enrichissant de façon cohérente le questionnement de l'histoire du genre. Son œuvre se caractérise par le fait qu'elle n'a jamais considéré la question du genre comme une alternative aux autres champs historiques. Ne pas catégoriser l'histoire du genre comme un domaine séparé de l'histoire économique et sociale mais « la thématiser comme composante centrale de la complexité de la société » (p.12), tel est le message central des articles rassemblés dans cet ouvrage.

Le livre est structuré en cinq parties thématiques et comprend quatorze articles rédigés par Karin Hausen entre 1976 et 2012. Notons que les textes ont été, pour la plupart, volontairement laissés dans leur forme d'origine. Aux articles publiés s'ajoute un texte original: une relecture de son célèbre article sur les marqueurs de genre («Geschlechtscharaktere »).

4 La première partie, intitulée "Bürgerliche Geschlechterordnung " (L'ordre sexué bourgeois), s'ouvre d'ailleurs sur cet article fondateur consacré à la polarisation des marqueurs de genre. Ce texte, entretemps devenu une référence, constituait à l'époque une révélation. Karin Hausen y décrit la formation d'un ordre sexué dont l'ancrage biologico-physique a été construit dès la fin du xvIII ${ }^{\mathrm{e}}$ siècle par la bourgeoisie montante. À partir de ce postulat, l'historienne déconstruit les oppositions « naturelles » hommefemme, qui ont donné une assise historique à la séparation genrée entre sphère publique et sphère privée. Dans la relecture de son article, elle explique que sa propre expérience en tant que jeune chercheuse et mère a joué un rôle certain dans la cristallisation de son intérêt pour la question de cette séparation genrée. En plus de ce rapprochement intéressant entre vie privée et vie scientifique, l'auteure répond aux critiques que son article avait suscitées en proposant une réflexion renouvelée sur l'interaction entre discours idéologico-normatifs et réalités sociales.

5 La deuxième partie, « Haushalt und Technik » (Ménage et technique), traite du « vide théorique entre l'économie domestique et l'économie capitaliste agro-industrielle » (p. 110). L'entretien du foyer par les femmes est ici soustrait à son enrobage « privé » et «traditionnel» pour être intégré dans le processus d'industrialisation et d'urbanisation. Dans ses études consacrées à l'histoire sociale de la machine à coudre, de la lessive ou de l'économie du bois de chauffage, Karin Hausen a su prendre en compte des objets souvent très concrets pour les interpréter comme prismes révélateurs de mutations socio-économiques plus profondes. Accompagné par le bourdonnement des machines à coudre et les vapeurs des chambres à lessive, le lecteur est plongé dans l'exécution des tâches domestiques, dans la division du travail et dans 
les effets des innovations techniques sur les conditions de vie et de travail des femmes au XIX ${ }^{e}$ siècle.

6 L'imbrication complexe entre ordres social, genré et économique est également abordée dans la troisième partie, " Arbeiten, Wirtschaften und Geschlechterdifferenz » (Occupations, économies et différence des sexes). Les articles rassemblés ici mettent en évidence la manière dont la répartition genrée des tâches a joué un rôle central dans le maintien d'une certaine stabilité sociale au $\mathrm{XIX}^{\mathrm{e}}$ siècle. L'auteure exemplifie ce processus par l'étude de mesures socio-politiques comme la protection de la maternité ou les assurances maladie au tournant du siècle. L'assignation des hommes et des femmes à des activités professionnelles bien déterminées et la valorisation inégale dont elles font l'objet sont présentées comme des tendances qui perdurent du début de l'époque moderne jusqu'à aujourd'hui.

7 La quatrième partie, « Muttertag, Volkstrauertag und andere Antworten auf den Ersten Weltkrieg » (Fête des mères, jour de deuil national et autres réponses à la Première Guerre mondiale), est consacrée aux commémorations en Allemagne après la Première Guerre mondiale, marquées par des représentations de genre. À travers des exemples comme le Volkstrauertag et la Fête des mères, l'historienne met en évidence les dispositifs ciblés de commercialisation et de médiatisation qui, en consacrant le soldat comme idéal masculin et l'épouse-mère comme modèle féminin, servent non seulement la cohésion nationale mais aussi des objectifs concrets de politiques de population. $\mathrm{Ce}$ procédé qui érigeait la mère en figure sacro-sainte si éloignée des préoccupations des vraies mères est l'objet du dernier texte du chapitre sur la situation des veuves de soldats dans la République de Weimar. Il s'agit ici d'une étude innovante dans laquelle les femmes sont présentées en tant que victimes de guerre et actrices à l'arrière.

8 Le recueil s'achève sur des réflexions plus générales relatives aux "Theoretische und historiographische Herausforderungen » (Défis théoriques et historiographiques). Dans le dernier article, Karin Hausen critique la hiérarchisation de certains concepts analytiques et interprétatifs dans la recherche historique actuelle et plaide pour l'intégration d'une forme constructive de discordances et d'hétérogénéités. Dans l'optique de ce programme prônant l'impossibilité d'une histoire unique et unifiée ("Nicht-Einheit der Geschichte»), l'auteure souhaiterait que la recherche prenne davantage en compte l'hétérogénéité du temps et de l'espace étudiés, ainsi que la complexité de l'humain en fonction de l'origine, de la situation de vie, de l'âge et du sexe. Le livre témoigne de l'inscription de Karin Hausen dans ce programme qu'elle a contribué à élaborer et qu'elle continue d'enrichir par son attention accordée aux dynamiques sociales jusque-là invisibles, ainsi qu'à leurs cassures et interfaces.

\section{AUTEURS}

\section{SARAH BAUMANN}

Zeitgeschichte. Université de Fribourg, Suisse 